УДК 62-83.004.15

Волошин А. И., Цыганаш В. Е.

\title{
ПЕРСПЕКТИВЫ СОВЕРШЕНСТВОВАНИЯ СИСТЕМЫ УПРАВЛЕНИЯ ДЛЯ ДСП-15
}

При разработке современной системы управления мощного энергопотребителя ДСП-15 отдано предпочтение непосредственно решаемой задаче и учтены два взаимно дополняющих друг друга аспекта [1].

Во-первых, целевая направленность. Мощный энергопотребитель спроектирован как система в соответствии с ее применением и поставленными перед ней целями на основе самой современной элементной базы с программным управлением. При этом обеспечивается соответствующая более высокая универсальность, позволяющая регулировать расстояния между электродами, для достижения более равномерного распределения температуры жидкого металла в ковше. Однако этому решению препятствуют два фактора: хорошо отработанная реализация конструкции крепления электродов и сравнительно большие запаздывания при отработке входных воздействий. Поэтому при целевой направленности с учетом потребностей на универсальность разрабатываемой системы приходится накладывать ограничения и как в случае сложной системы осуществлять некоторый баланс.

Во-вторых, аспект, ориентированный на универсализацию. При этом разработан такой алгоритм управления для системы, при котором приоритетными являются общность и расширяемость. Эти два понятия взаимно дополняют друг друга [1]. Поэтому целью разработки можно выбрать синтез адаптивной системы управления, реализующей заданный технологический алгоритм.

Успешного сочетания этих аспектов можно добиться, если для мощного энергопотребителя выбрать в качестве целевой функции коэффициент использования мощности источника питания $K_{n}(t)$. С его помощью можно полнее характеризовать структуру энергопотребителя как системы, учесть взаимодействие источника питания и нагрузки, включая энергоперетоки между ними. Для одностадийного преобразования энергии его удобно представить в виде:

$$
K_{n}(t)=\frac{P_{n}(t)}{P_{H}(t)}=\frac{P_{H}(t)-P_{n o m}(I)-\Delta P_{p}(t)}{P_{H}(t)},
$$

где $P_{H}(t)=U I$ - фактическая мощность источника питания, зависящая от тока $I$ и напряжения $U$, которая подведена к нагрузке;

$P_{\text {nот }}(I)$ - мощность потерь, зависящая от тока $I$ силовой цепи;

$P_{n}(t)$ - фактическая полезная мощность, выделяемая на нагрузке;

$\Delta P_{p}(t)=I \Delta U+U \Delta I-$ недоиспользованная мощность источника питания, возникающая в результате отклонения от оптимального режима по току на $\Delta I$ и по напряжению на $\Delta U$;

$t$ - время.

Очень важным достоинством такой реализации целевой функции является то, что в ее основе находится уравнение баланса мощностей, позволяющее получать объективную информацию о процессе без запаздывания, а наличие в составе аддитивных и мультипликативных составляющих, представляющих частные критерии $P_{t}(t), P_{n o m}(I), \Delta P_{n o m}(t)$, позволяет просто формировать из числителя составной критерий как в дифференциальной, так и в интегральной форме. А это, в свою очередь, открывает перспективу не только получения со- 
ставного критерия оптимального управления $K_{n}(t)$ для энергопотребителя в целом, но и дает основу для разработки математической модели системы [2]. Особенно он перспективен при решении задач динамики, так как при этом максимум функционала обеспечивается при максимуме подинтегральной функции.

Важной особенностью такого подхода является и то обстоятельство, что он хорошо подходит к задачам, имеющим очень высокий уровень неопределенности при получении информации о процессе. К этому типу задач относится и построение систем управления дуговыми сталеплавильными печами при использовании в их контуре управления трех регуляторов, работающих на один объект, и оптимизатора, в качестве которого может быть использован оператор (сталевар) - лицо, принимающее решения [3].

Таким образом, практическая необходимость исследований в этом направлении очевидна.

Целью настоящей работы являются вопросы анализа и синтеза, которые обеспечивают помимо минимума среднеквадратичного критерия качества также и стабильность замкнутой системы (т. е. сохранение устойчивости при малых отклонениях действительных значений параметров от расчетных), а также выполнение других практических требований к системе оптимального управления мощным энергопотребителем.

Обобщая рассмотренное в постановочной части статьи, отметим, что конечной целью разработки является синтез адаптивной системы управления, реализующей заданный технологический алгоритм и обеспечивающей стабильность ее работы.

В этом плане, как показывает практика, одним из наиболее эффективных критериев управления энергопотреблением может быть коэффициент использования мощности источника питания $K_{n}(t)$. Его связь с ключевыми параметрами технологического процесса открывает перспективу особо тесного взаимодействия с моделью.

Не менее важным является и то обстоятельство, что измерение и фильтрация, с одной стороны, и управление и регулирование, с другой стороны, находятся друг с другом в некоторой замечательной взаимосвязи, которая впервые была выяснена Калманом и сформулирована им как принцип двойственности [4].

Благодаря этому принципу по аналогии с механикой можно ввести относительную, абсолютную и переносную системы координат и для сигналов. Это открывает перспективу проведения декомпозиции для мощного энергопотребителя и представления его структуры в виде отдельных подсистем. Решение задачи декомпозиции может быть осуществлено по аналогии с ее решением для струйного насоса [5].

Такой подход значительно упрощает анализ системы в целом. В частности, это делает возможным и проведение анализа на устойчивость и стабильность такой системы, опираясь на ее модель, которая выполняет и функции анализатора для сигналов, характеризующих ток и напряжение энергопотребителя.

Ориентируясь на эти обстоятельства, проведем анализ структурных особенностей реализуемой системы управления для одноступенчатого преобразователя энергии. Начнем с наиболее доступных для точного измерения переменных - тока $I$ и напряжения $U$. С их помощью можно охарактеризовать структуру энергопотребителя как систему, содержащую две подсистемы ИП - источник питания и $\mathrm{H}$ - нагрузка. Математически их также можно представить как преобразователи $\frac{U^{2}}{Z_{1}}$ и $I^{2} Z_{2}$, где $U, I$ - действующие значения напряжения и тока в подсистемах ИП и Н соответственно, $Z_{1}, Z_{2}$ - полные сопротивления подсистем ИП и Н. Чтобы при таком представлении структуры системы проще учитывать динамику процессов, происходящих в ней, проведем ее агрегирование [6] и перейдем к единой определяющей переменной, - мощности. Это позволит полнее охарактеризовать структуру энергопотребителя в целом, учесть взаимодействие подсистем ИП и Н между собой и уточнить представление о связи между математической моделью и физической реальностью. 
Переход к новой определяющей переменной наряду с более полной характеристикой структуры энергопотребителя дает возможность учесть энергоперетоки между подсистемами. Этот переход подтверждает возможность единой математической декомпозиции системы на основе трех частных критериев $P_{n}(t), P_{n o m}(I), \Delta P_{p}(t)$. А это в свою очередь, открывает перспективу получения составного критерия оптимального управления $K_{n}(t)$ для энергопотребителя в целом и дает основу для разработки математической модели системы.

Переход к $K_{n}(t)$ также позволяет произвести сочетание двух регуляторов в системе: оптимального и обычного, синтезированного исходя из заданных показателей качества системы регулирования.

Благодаря последней особенности для существенных отличий конечных значений $K_{n}(t)$ от его начальных значений используется оптимальный закон управления, а в области малых отклонений от цели управления система работает в режиме слежения. При дальнейшей детализации структуры естественно потребовать, чтобы система в процессе эксплуатации сама автоматически получала и использовала для синтеза желаемого регулирования недостающую информацию. При этом она приобретает новое качество - качество самонастройки.

Применение принципов самонастройки (адаптации) по сравнению с неадаптивным управлением позволяет вскрыть и реализовать целый ряд новых возможностей системы, поэтому остановимся на этом подробнее. Сами процессы адаптации удобно рассматривать отдельно для установившихся режимов и для динамики. Важной составляющей, которую иногда необходимо корректировать по ходу работы системы, являются потери мощности. При автоматическом поддержании $\Delta P_{p}(t)=0$ это дает возможность формировать оптимальную программную траекторию работы системы. Если по ходу процесса возникает необходимость коррекции этой траектории, то эту операцию выполняет оптимизатор, функции которого возложены на оператора - лицо, принимающее решения (ЛПР). Необходимость такого вмешательства возникает при переходах с одного технологического или электрического режима работы энергопотребителя на другой. Обычно такая смена режима сопровождается значительными изменениями мощности, поступающей в систему, и обеспечивается изменением управляющего (задающего) воздействия и коррекцией выходных сигналов регуляторов объекта.

Характерной особенностью вносимых исправлений в работу системы управления мощным энергопотребителем является то, что они касаются только коррекции медленных движений в системе и поэтому не усложняют, а облегчают работу оптимизатора. Но их проведение оказывает влияние и на быстрые движения, источником которых в основном является контур самонастройки, внешние воздействия и возмущения.

В конечном счете, вмешательство оптимизатора (ЛПР) направлено на то, чтобы достигалось состояние устойчивого динамического равновесия между подсистемами ИП и Н. Математическим условием его достижения и поддержания является $K_{n}(t) \rightarrow \max$, $\Delta P_{p}(t)=0$ при заданном оператором значении управляющего воздействия. Для этого состояния также характерно, что влияние внешних воздействий, изменений параметров и помех в таком режиме стремится к минимуму. Математически поддержание этого состояния означает такое разделение (декомпозиция) дифференциального уравнения на две подсистемы, при котором процессы, характеризующие «быстрые» движения себя практически не проявляют и ими можно пренебречь.

Физически это состояние означает, что относительное движение в системе сведено к минимуму, несмотря на наличие переносного и абсолютного движений в подсистемах ИП и Н.

Если рассматривать ИП как «окружающую среду», Н как объект в этой среде, то по аналогии с живой природой это состояние отвечает наилучшей приспособительной реакции живого организма на изменения в окружающей среде. 
Экспериментальную проверку этих технических решений предполагается провести в два этапа. На первом этапе предлагается осуществить проверку эффективности самого принципа управления режимом работы печи непосредственно по мощности, а на втором проверить эффективность системы управления в полном объеме.

Такой подход к управлению энергопотребителем позволяет: обеспечить работоспособность системы в условиях широкого изменения динамических свойств объекта, повысить надежность системы, унифицировать отдельные регуляторы или блоки регуляторов и приспособить их для работы с различными видами однотипных объектов.

\section{ВЫВОДЫ}

Экспериментальная проверка и внедрение предлагаемых технических решений в промышленных условиях наиболее полно были реализованы на дуговой сталеплавильной печи ДСП-12 [7]. Достоинства предлагаемой системы управления проявились в следующем:

1. Лицо, принимающее решения, (пультовщик) может принимать более обоснованные и своевременные решения по ходу плавки, так как в информационном потоке, поступающем на пульт управления в период расплавления шихты, значительно ослабляется влияние помех. Особенно заметно это проявляется, если в качестве шихты использовать металлолом.

2. Реализованная система предоставляет возможность выбора и поддержания оптимального положения рабочей точки в таком сложном трехфазном агрегате без нулевого провода, каким является ДСП.

3. Эффективность от внедрения модернизированной системы управления проявилась в том, что после внедрения системы удалось сократить расход электроэнергии на $10 \ldots 15$ тыс. кВт-час в месяц. Кроме того, в разы были сокращены поломки электродов.

\section{СПИСОК ИСПОЛЬЗОВАННОЙ ЛИТЕРАТУРЫ}

1. Системы: декомпозищия, оптимизация и управление / Сост. : М. Сингх, А. Титли ; Сокр. пер. с англ. A. В. Запорожиа. - М. : Машиностроение, 1986. - 496 с.

2. Цыганаш В. Е. Анализ модели силовой электрической иеепи мощчной электротермической установки / В. Е. Цыганаш // Наукові праці Донецького начіонального технічного університету. Серія : Обчислювальна техніка та автоматизація. - Донеиьк, 2003. - Вип. 58. - С. 130-135.

3. Цыганаш В.Е. Анализ и решение задачи выбора предпочтительной инициативы при управлении мошной электротермической установкой / В. Е. Цыганаш // Искусственный интеллект. - 2010. - № 2. C. $164-168$.

4. Браммер К. Фильтр Калмана-Бьюси / К. Браммер, Г. Зиффлинг ; пер. с нем. В. Б. Колмановского. M. : Наука. 1982. - 200 c.

5. Цыганаш В. Е. Постановка задачи оптимального управления проиессом создания и поддержания вакуума с помощью струйного насоса / В. Е. Цыганаш, А. И. Волошин // Вестник национального технического университета „ХПИ” „Новые решения в современных технологиях”: сб. науч. тр. - 2001. - Bыл. 14. - C. 206-212.

6. Первозванский А. А. Декомпозищия, агрегирование и приближенная оптимизация / А. А. Первозванский, В. Г. Гайцчгори. - М. : Наука, 1979. - 342 c.

7. Цыганаш В. Е. Частотный метод оптимального управления мощными энергопотребителями / В. Е. Цыганаш // Металлургическая и горнорудная промышленность. - 2009. - № 2. - С. 120-123. 